\title{
Relationship between sleep duration and self-reported health- related quality of life among US adults with or without major chronic diseases, 2014
}

\author{
Yong Liu, MD, MS ${ }^{\star}$, Anne G Wheaton, PhD, Janet B Croft, PhD, Fang Xu, PhD, Timothy J \\ Cunningham, ScD, SM, and Kurt J Greenlund, PhD \\ Division of Population Health, National Center for Chronic Disease Prevention and Health \\ Promotion, Centers for Disease Control and Prevention, Atlanta, USA
}

\begin{abstract}
Objectives-To assess the association between sleep duration and health-related quality of life (HRQOL) among adults with or without chronic conditions.

Methods-Using the 2014 Behavioral Risk Factor Surveillance System, we analyzed selfreported data from adult respondents aged $\geq 18$ years with $(n=277,757$, unhealthy group) and without ( $n=172,052$. healthy group) reported history of any of nine chronic conditions (coronary heart disease, stroke, cancer, chronic obstructive pulmonary disease, diabetes, asthma, arthritis, depression, chronic kidney disease). Multivariable logistic regressions were separately constructed to assess the associations between sleep duration and four self-reported HRQOL measures after adjustment for sociodemographics, leisure-time physical activity, body mass index, and smoking status among unhealthy and healthy adults.
\end{abstract}

Results-The prevalence of poor/fair health, frequent physical distress, frequent mental distress, frequent activity limitation, and short sleep duration was $27.9 \%, 19.3 \%, 17.0 \%, 13.6 \%$, and $38.3 \%$ in the unhealthy group and $6.9 \%, 4.0 \%, 5.3 \%, 2.1 \%$, and $31.0 \%$ in the healthy group, respectively. U-shaped relationships of sleep duration to all four HRQOL indicators were observed among the unhealthy group and to poor/fair health, frequent mental distress, and frequent activity limitation among the healthy group. The relationships further varied by sex, age, race/ethnicity, and BMI category among the healthy group.

Conclusions-Relationships between extreme sleep duration and HRQOLs were observed among both healthy and unhealthy groups. These results can help inform public awareness campaigns and physician-counseling regarding the importance of sleep for mental health and wellbeing.

\footnotetext{
Disclaimer: The conclusions, findings, and opinions expressed by authors do not necessarily reflect the official position of the Centers for Disease Control and Prevention.

*Corresponding author at: Division of Population Health, National Center for Chronic Disease Prevention and Health Promotion, Centers for Disease Control and Prevention, 4770 Buford Highway, MS F-78, Atlanta, GA 30341. IKD8@ CDC.GOV (Y. Liu). Supplementary data to this article can be found online at https://doi.org/10.1016/j.sleh.2018.02.002.

Conflict of interest The authors have nothing to disclose.
} 


\section{Keywords}

Health-related quality of life; Sleep; Population-based survey; Cross-sectional study; Mental health; Activity limitations

\section{Introduction}

Health-related quality of life (HRQOL), a multi-dimensional measure that includes areas of physical, mental, emotional, and social functioning (https://www.cdc.gov/hrqol/ concept.htm), provides a reliable approach to assess an individual's health status. Prior studies indicate that lower HRQOL is associated with chronic disease, short and long-term disabilities, and sleep health. ${ }^{1-4}$

Sleep is an essential daily behavior. Sleep disorders such as restless legs syndrome and insomnia are known to impact patients' quality of life,, 5 but recent studies have suggested that sleep duration may also be an important predictor of an individual's health status. For example, sleep duration, particularly short sleep duration ( $<7$ hours sleep in a 24-hour period), may be associated with sociodemographics, mental health, risk behaviors, and chronic diseases. ${ }^{7-15}$ Emerging evidence from longitudinal studies suggests that short sleep duration is more likely to be associated with chronic disease than sufficient sleep duration ( $\geq 7$ hours/24 hours). ${ }^{14}$ A U-shaped relationship was reported between sleep duration and self-rated health and quality of life among middle-aged and elderly Australians in one study. ${ }^{16}$ Short sleep duration was found to be associated with poor self-rated health among college students aged 17-30 years in another study. ${ }^{17}$ However, research on the relationship between sleep duration and HRQOL is scarce.

Since both sleep duration and HRQOL are associated with chronic conditions, we hypothesized that the relationship between sleep duration and HRQOL in the healthy group without the nine chronic conditions would be different from that in the unhealthy group. We sought to assess the relationship between sleep duration and HRQOL among healthy adult respondents without a history of any of nine chronic conditions separately from adults with a history of any of those conditions. Furthermore, we examined the relationship among adults without any of the chronic conditions in groups defined by sex, age, race/ethnicity, and body mass index (BMI) categories.

\section{Methods}

The Behavioral Risk Factor Surveillance System (BRFSS) is an ongoing, state-based, random-digit-dialed telephone survey of non-institutionalized persons aged 18 years or older in 50 states, the District of Columbia, and territories, which is supported by the Centers for Disease Control and Prevention. In 2014, BRFSS collected data on sleep duration, HRQOL, health-risk behaviors, chronic conditions, and sociodemographic characteristics. The survey sample was selected through a multistage sampling design of households with either landline or cellular telephones. Response rates for BRFSS are calculated using standards set by the American Association of Public Opinion Research (AAPOR) Response Rate Formula \#4 (https://www.aapor.org/AAPOR_Main/media/publications/Standard- 
Definitions20169theditionfinal.pdf). The response rate is the number of respondents who completed the survey as a proportion of all eligible and likely-eligible persons. The median survey response rate from both landline and cellular phone interviews for all 50 states, the District of Columbia, and territories in 2014 was $47.0 \%$ and ranged from $25.1 \%$ to $60.1 \%$ (http://www.cdc.gov/brfss/annual_data/2014/pdf/2014_DQR.pdf). Additional information about the 2014 survey is available (http://www.cdc.gov/brfss/annual_data/ annual_2014.html).

The unhealthy group was defined as adults with any of nine chronic conditions (coronary heart disease [CHD], stroke, cancer, chronic obstructive pulmonary disease [COPD], diabetes, asthma, arthritis, depression, chronic kidney disease). The healthy group was defined as adults without any of the nine chronic conditions. Chronic disease status was determined based on responses to several questions. Respondents were asked whether a health professional had ever told them they had any of a series of conditions. CHD was defined as "heart attack also called a myocardial infarction" or "angina or coronary heart disease." Cancer included skin cancer or any other type of cancer. COPD was defined as "chronic obstructive pulmonary disease or COPD, emphysema, or chronic bronchitis." Diabetes included respondents who had ever been told they had diabetes, but did not include gestational diabetes, borderline diabetes, or pre-diabetes. Arthritis included any "form of arthritis, rheumatoid arthritis, gout, lupus, or fibromyalgia." Depression was defined as "a depressive disorder, including depression, major depression, dysthymia, or minor depression." For chronic kidney disease, respondents were asked if they had been told they had kidney disease, but not to include kidney stones, bladder infection, or incontinence.

Sleep duration was categorized into $5,6,7$ (referent group), 8, 9, $\geq 10$ hours based on a response to "On average, how many hours of sleep do you get in a 24-hour period?" Short sleep duration was defined as those who reported $<7$ hours.

The validity and reliability of the four individual-level HRQOL questions were previously established for population health surveillance. ${ }^{18}$ Self-rated health was assessed by asking respondents to rate their health status on a five-point scale from excellent to poor, which was dichotomized into poor/fair vs. good/very good/excellent. The other three HRQOL questions about physical health, mental health, and activity limitation were: "Now thinking about your physical health, which includes physical illness and injury, for how many days during the past 30 days was your physical health not good?", "Now thinking about your mental health, which includes stress, depression, and problems with emotions, for how many days during the past 30 days was your mental health not good?", and "During the past 30 days, for about how many days did poor physical or mental health keep you from doing your usual activities, such as self-care, work, or recreation?" Responses to each question were dichotomized into $<14$ (referent group) and $\geq 14$ (defined as unfavorable HRQOL) unhealthy days. As in previous research, frequent physical distress was defined as $\geq 14$ physically unhealthy days, frequent mental distress was defined as $\geq 14$ mentally unhealthy days, and frequent activity limitation was defined as $\geq 14$ days of activity limitation. ${ }^{19}$ HRQOL measures were analyzed separately in our study because these measures reflected different aspects of health status. In addition, there was only a small or moderate correlation between the HRQOL measures (Pearson's correlation coefficient ranged from 0.2 to 0.6 ). 
Covariates included age group (18-24, 25-34, 35-44, 45-64, or $\ 65$ years), sex, race/ ethnicity (non-Hispanic White, non-Hispanic Black, Hispanic, non-Hispanic American Indian/Alaska Native, non-Hispanic Asian, or non-Hispanic other, which included Native Hawaiian/Pacific Islander, multiracial, or other), education (less than high school, high school diploma or GED, some college or technical school, or college graduate), marital status (married; divorced, separated, or widowed; never married; or a member of an unmarried couple), employment status (employed, unemployed, unable to work, retired, or homemaker or student), and household income (categorized as $0-<\$ 25,000, \$ 25,000-<$ $\$ 50,000, \geq \$ 50,000$, or missing, due to the large proportion of respondents who did not report their income). There was no collinearity of education, employment, and household income, so these three socio-economic variables were included in the analysis. Any leisure-time physical activity was defined based on the response of 'yes' or 'no' to a single question "During the past month, other than your regular job, did you participate in any physical activities or exercises such as running, calisthenics, golf, gardening, or walking for exercise?" BMI was calculated from self-reported height and weight (underweight: $\mathrm{BMI}<18.5 \mathrm{~kg} / \mathrm{m}^{2}$, normal weight: $\mathrm{BMI}=18.5-24.9 \mathrm{~kg} / \mathrm{m}^{2}$, overweight: $\mathrm{BMI}=25.0-29.9$ $\mathrm{kg} / \mathrm{m}^{2}$, or obese: BMI $\geq 30.0 \mathrm{~kg} / \mathrm{m}^{2}$ ). Smoking status was defined by two questions: "Have you smoked at least 100 cigarettes in your entire life?" and "Do you currently smoke every day, some days, or not at all?" Respondents were current smokers if they reported having smoked at least 100 cigarettes during their lifetime and currently smoke every day or some days. Former smokers were defined as those who reported having smoked at least 100 cigarettes during their lifetime but did not currently smoke. Never smokers were defined as those who reported not having smoked at least 100 cigarettes during their lifetime.

Of 464,664 adult respondents who participated in the 2014 BRFSS survey, 286,381 adult respondents $(52.1 \%$, unhealthy group) reported a history of any of the nine chronic conditions (CHD, stroke, cancer, COPD, diabetes, asthma, arthritis, depression, chronic kidney disease) and 176,558 adult respondents (47.9\%, healthy group) reported none of the nine chronic conditions. Because of significant interactions between sleep duration and chronic conditions $(\mathrm{p}<0.1)$, we separately analyzed data on the unhealthy group $(\mathrm{n}=$ $277,757)$ and the healthy group $(\mathrm{n}=172,052)$ after excluding those who had missing values on age, sleep duration, and self-reported health status $(n=8,624$ in unhealthy group and $n=$ 4,506 in healthy group).

\section{Statistical analysis}

First, we calculated the distribution of selected characteristics including sociodemographics, risk factors, sleep duration, and HRQOL in the healthy and unhealthy groups. Second, the crude and age-adjusted prevalence of four HRQOL measures and short sleep duration were assessed by selected characteristics among the healthy group and the unhealthy group separately. Statistical significance for two-tailed t-test was set at $p<0.05$. Then, multivariable logistic regression models were constructed to assess relationships between sleep duration and the four HRQOL measures after adjustment for sociodemographics, leisure-time physical activity, BMI category, and smoking status among the healthy group and the unhealthy group separately. Finally, because sex, age, race/ethnicity, and BMI category were main confounding factors on sleep duration and HRQOL, fully-adjusted logistic regression 
models stratified by sex, age group, race/ethnicity, and BMI category were conducted only among the healthy group to further assess the relationship between sleep duration and HRQOL. Only race/ethnicity groups with sufficient sample size (non-Hispanic White, nonHispanic Black, and Hispanic) were used for stratified analysis. All analyses were conducted by using SAS-callable SUDAAN (Release 11.0.1, Research Triangle Institute, Research Triangle Park, NC) to account for the complex study design and sampling weights. BRFSS sampling weights adjust for noncoverage and nonresponse and provide estimates to be representative of the civilian, non-institutionalized adult population aged $\geq 18$ years in the US (https://www.cdc.gov/brfss/data_documentation/pdf/UserguideJune2013.pdf).

\section{Results}

The distribution of many characteristics differed significantly between unhealthy and healthy adult respondents (Table 1). Compared to the unhealthy group, respondents in the healthy group were more likely to be men, younger, Hispanic or non-Hispanic Asian, college graduates, have a household income $\$ \$ 50,000$, employed or homemaker or student, never married, never smokers, normal weight, engage in leisure-time physical activity, and usually sleep 7 or 8 hours $(P<.001)$. The prevalence of each unfavorable HRQOL variable was much lower among adult respondents with none of the nine chronic conditions than respondents with at least one.

Age-adjusted percentages of four unfavorable HRQOL measures and short sleep duration by selected characteristics among unhealthy adults with at least one of nine chronic conditions are shown in Table 2. Women reported a higher prevalence of the four HRQOL measures than men $(P<.001)$, with no significant difference in short sleep duration. Adults aged 1824 years had a lower prevalence of poor or fair health, frequent physical distress, and frequent activity limitation than older age groups. Adults aged 25-34 years had a higher prevalence of frequent mental distress and adults aged 45-64 years had a higher prevalence of poor or fair health, frequent physical distress, and frequent activity limitation than other age groups. Hispanics had a higher prevalence of poor or fair health and American Indians/ Alaska Natives had a higher prevalence of frequent physical distress and frequent mental distress than other racial/ethnic groups. Non-Hispanic Asians had the lowest prevalence of the four HRQOL measures and non-Hispanic Whites had a lower prevalence of short sleep duration than the other groups. There was an inverse relationship between education level, household income, and the four unfavorable HRQOL measures and short sleep duration. Adults who were unable to work had the highest prevalence of the four unfavorable HRQOL measures and short sleep duration, while employed persons had the lowest prevalence of the four unfavorable HRQOL measures and homemakers or students had the lowest prevalence of short sleep duration. Married persons had the lowest prevalence of the four unfavorable HRQOL measures, while persons who were divorced, widowed, or separated had the highest prevalence of the four unfavorable HRQOL measures and short sleep duration. A U-shaped relationship between BMI category and the four unfavorable HRQOL measures and short sleep duration was observed. Current smokers and adults reporting no-leisure-time physical activity had a higher prevalence of the four HRQOL measures and short sleep duration than their counterparts, while never smokers had a lower prevalence of the four HRQOL measures and short sleep duration than their counterparts. 
Age-adjusted percentages of four unfavorable HRQOL measures and short sleep duration by selected characteristics among adults with no known chronic conditions are shown in Table 3. Men were more likely than women to report having poor or fair health and short sleep duration, while women were more likely to report frequent mental distress $(P<.001)$. Adults aged 18-24 years had a lower prevalence of poor or fair health and a higher prevalence of frequent mental distress than older age groups. Adults aged $₫ 65$ years had a lower prevalence of frequent mental distress and short sleep duration than younger age groups. Hispanics had a higher prevalence of poor or fair health and frequent physical distress than other racial/ethnic groups. Non-Hispanic Whites had a lower prevalence of poor or fair health than the other groups. Non-Hispanic Blacks had a higher prevalence of short sleep duration than their counterparts. There was an inverse relationship between education level, household income, and the four unfavorable HRQOL measures, whereas persons with a high school diploma or some college had a higher prevalence of short sleep duration than their counterparts. Adults who were unable to work had the highest prevalence of the four unfavorable HRQOL measures, and employed persons had the lowest prevalence of frequent physical distress and frequent activity limitation. Married persons had the lowest prevalence of poor or fair health, frequent physical distress, and frequent mental distress while members of an unmarried couple had the highest prevalence of poor or fair health. In addition, persons who were divorced, widowed, or separated had the highest prevalence of frequent mental distress, frequent activity limitation, and short sleep duration. A U-shaped relationship between BMI category and the four unfavorable HRQOL measures and short sleep duration was observed. Current smokers and adults with no-leisure-time physical activity had a higher prevalence of the four HRQOL measures and short sleep duration than their counterparts while, never smokers had a lower prevalence of short sleep duration than their counterparts.

U-shaped relationships of sleep duration to the prevalence of all four unfavorable HRQOL indicators were observed among unhealthy adults with nine chronic conditions and healthy adults without the nine chronic conditions (Table 4). The U-shaped relationship between sleep duration and HRQOL indicators persisted among unhealthy adults after adjustment for sociodemographics and health-risk behaviors for all four indicators. Compared to unhealthy adults who slept 7 hours, those who reported shorter or longer sleep durations were more likely to report frequent physical distress and frequent activity limitation, while those who slept $<7$ or $>8$ hours were more likely to report poor or fair health and frequent mental distress $(P<.001)$. Among healthy adults, the U-shaped relationships persisted in fully adjusted models for three of the four indicators. Compared to healthy adults who slept 7 hours, those who slept $\leq$ hours, 6 hours, or $\geq 10$ hours were significantly more likely to report poor or fair health (adjusted prevalence ratio (PR) $[95 \%$ confidence interval $]=1.70$ [1.50-1.94], 1.37 [1.22-1.53], and 1.34 [1.04-1.74], respectively), frequent mental distress (2.85 [2.49-3.27], 1.76 [1.55-1.99], and 1.65 [1.20-2.27], respectively), and frequent activity limitation (2.68 [2.13-3.36], 1.68 [1.36-2.08], and 2.35 [1.43-3.89], respectively). Frequent physical distress was significantly more likely to be reported in those who slept $\mathbf{5}$ hours or 6 hours (1.76 [1.48-2.10] and 1.22 [1.05-1.42], respectively), but was no longer significant among those who slept $\geq 10$ hours in the fully-adjusted model. 
The relationship between sleep duration and all four individual HRQOL indicators among adults without any of the nine chronic conditions varied by sex, age, race/ethnicity, and BMI categories in fully-adjusted logistic regression models (Tables S1-S4). A U-shaped relationship between sleep duration and poor or fair health was observed among men, while a higher risk of reporting poor or fair health was observed only among women who reported shorter sleep durations (Table S1). In contrast, a U-shaped relationship between sleep duration and frequent physical distress was observed among women, while a higher risk of reporting frequent physical distress was observed only among men who reported sleeping $\mathbf{5}$ hours. AU-shaped relationship between sleep duration and frequent mental distress was observed among men, while a higher risk of reporting frequent mental distress was observed only among women who reported shorter sleep durations. A U-shaped relationship between sleep duration and frequent activity limitation was observed among both men and women.

The risk for poor or fair health was significantly higher only for those who slept $\geq 10$ hours among adults aged 18-24 years, while there was a U-shaped relationship among adults aged 265 years (Table S2). Among adults in the intermediate age groups, an increased risk for poor or fair health was observed with short sleep durations only. Sleep duration was not associated with frequent physical distress among adults in the youngest age group. In the other age groups, a higher risk for frequent physical distress was observed with short sleep duration only. A U-shaped relationship was observed between sleep duration and frequent mental distress among adults aged 25-34 or 35-44 years, with an increased risk only for short sleep duration among younger or older adults. A U-shaped relationship was observed between sleep duration and frequent activity limitation among adults aged 25-34 years or aged 45-64 years, with an increased risk only for short sleep duration in other age groups.

A U-shaped relationship was observed between sleep duration and poor or fair health among non-Hispanic white adults, with an increased risk for shorter sleep durations only among non-Hispanic Black and Hispanic adults (Table S3). Among all three race/ethnicity groups, an increased risk of frequent physical distress was higher for short sleep durations only. An increased risk for frequent mental distress was observed for short sleep durations among non-Hispanic White and Hispanic adults; however, a significant U-shaped relationship with sleep duration was observed among non-Hispanic Black adults. For frequent activity limitation, an increased risk was observed for short sleep durations among non-Hispanic White and Black adults and a significant U-shaped relationship with sleep duration among Hispanic adults.

An increased risk for poor or fair health was observed for short sleep durations among normal weight and obese adults; however, a significant U-shaped relationship with sleep duration was observed among overweight adults (Table S4). There was no association among underweight adults. The risk for frequent physical distress was higher only for short sleep durations among normal weight, overweight, and obese adults, with no association among underweight adults. The risk for frequent mental distress was higher for shorter sleep durations among all BMI categories. A U-shaped relationship between sleep duration and frequent activity limitation was seen among normal weight adults, but only short sleep durations presented an increased risk among the other three BMI categories. 


\section{Discussion}

Our results show significant $U$-shaped relationships between sleep duration and all four HRQOL measures among adults with a history of any of nine chronic conditions after adjustment for sociodemographics and health-risk behaviors. Similar significant U-shaped relationships were observed between sleep duration and poor or fair health, frequent mental distress, and frequent activity limitation among adults without a history of any of the nine chronic conditions. The relationships varied by sex, age, race/ethnicity, and BMI category in stratified analyses in the healthy group. For nearly all strata defined by sex, age group, racial/ethnic group, or BMI group, the increased risk of reporting unfavorable HRQOL indicators remained for shorter sleep durations. The increased risk with longer sleep durations disappeared for most HRQOL indicators for men and women and some age, racial/ethnic, or BMI groups.

The results from previous research on the relationships between sleep duration and HRQOL indicators have been mixed. A previous study that demonstrated a U-shaped relationship between sleep duration and self-rated poor or fair health among US adults aged $\geq 18$ years found that the U-shaped relationship persisted in most subgroups: men, women, nonHispanic Whites, non-Hispanic Blacks, non-obese, and obese adults. ${ }^{20} \mathrm{~A}$ study of older adults in Spain found a U-shaped relationship between sleep duration and the physical summary component of the SF-36 questionnaire among women, with only short sleep duration being associated with worse scores on the mental scale. ${ }^{3}$ That study found no association between sleep duration and the physical and mental scales among men. Among Australians aged 45-84 years, U-shaped relationships were observed between sleep duration and self-rated health and quality of life. ${ }^{16}$ When stratified by age, the U-shaped associations remained for all but the oldest age group. Among Korean adults aged 18 years and over, a Ushaped relationship between sleep duration and HRQOL using the EuroQOL-5 Dimension was observed among both men and women. ${ }^{21}$ Steptoe et al. reported that short sleep duration, but not long sleep duration, was associated with self-rated poor health compared to those who slept 7-8 hours among European college students aged $17-30$ years. ${ }^{17}$ Not all studies have found an association between sleep duration and HRQOL. In a cross-sectional study of San Diego residents aged 40-64 years $(n=273)$ for whom sleep duration was assessed from subjective questionnaire and actigraphy, sleep duration was not associated with well-being. ${ }^{22}$ Most findings in previous studies were based on adjustment for varying chronic conditions as confounders $3,16,17,20$; however, our results showed that significant $U$ shaped relationships of sleep duration with all four unfavorable HRQOL indicators remained among unhealthy adults and with three unfavorable HRQOL indicators among healthy adults, even after adjustment for sociodemographic factors and health-risk behaviors.

Although this is the largest phone interview survey to date to address the relationship between sleep duration and self-reported HRQOL, several limitations should be noted. First, because the BRFSS is a cross-sectional study, it is not possible to make causal inferences and determine whether short or long sleep duration result in poor health or HRQOL or vice versa. It is not possible to determine the degree to which short or long sleep duration is the cause of poor health or HRQOL or a marker of poor health, although other studies have provided some potential evidence for a causal association between short sleep duration, but 
not long sleep duration, and several chronic conditions. ${ }^{14}$ The results from our stratified analyses by sex, age, race/ethnicity, and BMI category support this hypothesis and the relationship between long sleep duration and HRQOL observed in some studies may be caused by confounding factors. Second, sample selection bias due to the low response rate may also impact our results. However, the effect of potential systematic bias is likely limited as the moderate relationship between sleep duration and unfavorable HRQOL revealed in our study is consistent with results from previous studies. Third, self-reported sleep duration on national surveillance systems has not been validated by polysomnography or actigraphy and may not be as accurate as these objective measures and may affect the results in the study. Finally, we defined our healthy sample based on the absence of nine chronic conditions, but other health conditions not included in BRFSS, such as sleep apnea, HIV, or inflammatory bowel disease, may affect HRQOL. ${ }^{23}$ Nearly 3000 adults in our healthy group reported that they were unable to work and may be suffering from conditions that were not included in our analyses.

\section{Conclusions}

We found that short sleep duration was associated with impaired HRQOLs even in the absence of many common chronic conditions. Strategies to increase the proportion of adults getting enough sleep may include continued public awareness campaigns, as well as improvement of education of non-sleep specialists on the role of sleep in their patients' health and how to advise their patients to improve their sleep. More research is needed on effective means of changing individuals' behaviors to improve sleep, including workplace interventions.

\section{Supplementary Material}

Refer to Web version on PubMed Central for supplementary material.

\section{References}

1. Allen NB, Badon S, Greenlund KJ, Huffman M, Hong Y, Lloyd-Jones DM. The association between cardiovascular health and health-related quality of life and health status measures among U.S. adults: a cross-sectional study of the National Health and Nutrition Examination Surveys, 20012010. Health Qual Life Outcomes. 2015; 13:152.doi: 10.1186/s12955-015-0352-z [PubMed: 26396070]

2. Zahran HS, Kobau R, Moriarty DG, Zack MM, Holt J, Donehoo R. Health-related quality of life surveillance-United States, 1993-2002. MMWR. 2005; 54(SS04):1-35.

3. Faubel R, Lopez-Garcia E, Guallar-Castillón P, et al. Sleep duration and health-related quality of life among older adults: a population-based cohort in Spain. Sleep. 2009; 32(8):1059-1068. [PubMed: 19725257]

4. Liu Y, Wheaton AG, Chapman DP, Croft JB. Sleep duration and chronic diseases among U.S. adults age 45 years and older: evidence from the 2010 Behavioral Risk Factor Surveillance System. Sleep. 2013; 36(10):1421-1427. DOI: 10.5665/sleep.3028 [PubMed: 24082301]

5. Morgenthaler T, Kramer M, Alessi C, et al. Standards of Practice Committee of the American Academy of Sleep Medicine. Practice parameters for the psychological and behavioral treatment of insomnia: an update. An American Academy of Sleep Medicine report. Sleep. 2006; 29(11):14151419. [PubMed: 17162987] 
6. Stevens MS. Restless legs syndrome/Willis-Ekbom disease morbidity: burden, quality of life, cardiovascular aspects, and sleep. Sleep Med Clin. 2015; 10(3):369-373. https://doi.org/10.1016/ j.jsmc.2015.05.017 [xv-xvi]. [PubMed: 26329447]

7. Gangwisch JE, Malaspina D, Boden-Albala B, Heymsfield SB. Inadequate sleep as a risk factor for obesity: analyses of the NHANES I. Sleep. 2005; 28:1289-1296. [PubMed: 16295214]

8. Gottlieb DJ, Punjabi NM, Newman AB, et al. Association of sleep time with diabetes mellitus and impaired glucose tolerance. Arch Intern Med. 2005; 165:863-867. [PubMed: 15851636]

9. Magee CA, Kritharides L, Attia J, McElduff P, Banks E. Short and long sleep duration are associated with prevalent cardiovascular disease in Australian adults. J Sleep Res. 2012; 21:441447. [PubMed: 22211671]

10. Schoenborn, CA., Adams, PF. Sleep duration as a correlate of smoking, alcohol use, leisure-time physical inactivity, and obesity among adults: United States, 2004-2006. National Center for Health Statistics2008Available from http://www.cdc.gov/nchs/data/hestat/sleep04-06/ sleep04-06.htm

11. Stamatakis KA, Kaplan GA, Roberts RE. Short sleep duration across income, education, and race/ ethnic groups: population prevalence and growing disparity during 34 years of follow-up. Ann Epidemiol. 2007; 17(12):948-955. [PubMed: 17855122]

12. Sabanayagam C, Shankar A. Sleep duration and cardiovascular diseases: results from the National Health Interview Survey. Sleep. 2010; 33:1037-1042. [PubMed: 20815184]

13. Van Mill JG, Hoogendijk WJ, Vogelzangs N, van Dyck R, Peninx BW. Insomnia and sleep duration in a large cohort of patients with major depressive disorders and anxiety disorders. J Clin Psychiatry. 2010; 71:239-246. [PubMed: 20331928]

14. Watson NF, Badr MS, Belenky G, et al. Consensus Conference Panel. Joint consensus statement of the American Academy of Sleep Medicine and Sleep Research Society on the recommended amount of sleep for a healthy adult: methodology and discussion. Sleep. 2015; 38:1161-1183. [PubMed: 26194576]

15. Yaggi HK, Araujo AB, McKinlay JB. Sleep duration as a risk factor for the development of type 2 diabetes. Diabetes Care. 2006; 29:657-661. [PubMed: 16505522]

16. Magee CA, Caputi P, Iverson DC. Relationship between self-rated health, quality of life and sleep duration in middle aged and elderly Australians. Sleep Med. 2011; 12:346-350. [PubMed: 21388876]

17. Steptoe A, Peacey V, Wardle J. Sleep duration and health in young adults. Arch Intern Med. 2006; 166:1689-1692. [PubMed: 16983045]

18. Andresen EM, Catlin TK, Wyrwich KW, Jackson-Thompson J. Retest reliability of surveillance questions on health related quality of life. J Epidemiol Community Health. 2003; 57(5):339-343. [PubMed: 12700216]

19. Shockey, TM., Zack, M., Sussell, A. Health-related quality of life among US workers: variability across occupation groups; Am J Public Health2017e1-e8

20. Shankar A, Charumathi S, Kalidindi S. Sleep duration and self-rated health: the National Health Interview Survey 2008. Sleep. 2011; 34(9):1173-1177. [PubMed: 21886354]

21. Kim J, Park E, Yoo K, Park S. The association between short or long sleep times and quality of life (QOL): results of the Korea National Health and Nutrition Examination Survey (KNHANES IVV). J Clin Sleep Med. 2015; 11(6):625-634. [PubMed: 25979101]

22. Jean-Louis G, Kripke DF, Ancoli-Israel S. Sleep and quality of well-being. Sleep. 2000; 23:11151121. [PubMed: 11145326]

23. Baldwin CM, Griffith KA, Nieto FJ, O'Connor GT, Walsleben JA, Redline S. The association of sleep-disordered breathing and sleep symptoms with quality of life in the Sleep Heart Health Study. Sleep. 2001; 24(1):96-105. [PubMed: 11204058] 


\section{Table 1}

The distribution of selected characteristics among adults aged $\geq 18$ years with or without any of nine chronic conditions $^{a}$, 2014 BRFSS

\begin{tabular}{|c|c|c|c|c|}
\hline \multirow[b]{2}{*}{ Characteristic } & \multicolumn{2}{|c|}{$\begin{array}{l}\text { Unhealthy group } \\
\text { Respondents with at least one of nine } \\
\text { chronic conditions }(\mathrm{n}=277,757)\end{array}$} & \multicolumn{2}{|c|}{$\begin{array}{l}\text { Healthy group } \\
\text { Respondents without any of nine chronic } \\
\text { conditions }(n=172,052)\end{array}$} \\
\hline & $\mathbf{N}^{b}$ & $\%(95 \% \mathrm{CI})^{c}$ & $\mathrm{~N}^{b}$ & $\%(95 \% \mathrm{CI})^{c}$ \\
\hline \multicolumn{5}{|l|}{ Sex } \\
\hline Men & 107,131 & $44.6(44.2-44.9)$ & 80,575 & $53.3(52.8-53.8)$ \\
\hline Women & 170,626 & $55.4(55.1-55.8)$ & 91,477 & $46.7(46.2-47.2)$ \\
\hline \multicolumn{5}{|l|}{ Age (years) } \\
\hline $18-24$ & 7,805 & $7.9(7.6-8.1)$ & 16,070 & $18.8(18.4-19.2)$ \\
\hline $25-34$ & 15,246 & $11.3(11.0-11.6)$ & 27,829 & $24.3(23.9-24.7)$ \\
\hline $35-44$ & 21,872 & $12.4(12.1-12.7)$ & 31,476 & $20.9(20.6-21.3)$ \\
\hline $45-64$ & 107,485 & $38.6(38.3-39.0)$ & 67,694 & $28.5(28.1-28.9)$ \\
\hline$\checkmark 65$ & 125,349 & $29.8(29.5-30.1)$ & 28,983 & $7.4(7.3-7.6)$ \\
\hline \multicolumn{5}{|l|}{ Race/Ethnicity } \\
\hline White $^{d}$ & 222,019 & $70.3(69.9-70.7)$ & 125,278 & $57.6(57.2-58.1)$ \\
\hline Black $^{d}$ & 20,187 & $11.2(10.9-11.5)$ & 13,126 & $11.9(11.6-12.2)$ \\
\hline Hispanic & 16,980 & $12.6(12.3-12.9)$ & 17,752 & $20.6(20.2-21.0)$ \\
\hline American Indian/Alaska Native ${ }^{d}$ & 4,258 & $1.1(1.0-1.2)$ & 2,557 & $0.8(0.8-0.9)$ \\
\hline $\operatorname{Asian}^{d}$ & 3,114 & $2.8(2.5-3.0)$ & 5,887 & $7.1(6.8-7.5)$ \\
\hline Other ${ }^{d}$ & 7,287 & $2.0(1.9-2.1)$ & 4,861 & $1.8(1.7-2.0)$ \\
\hline \multicolumn{5}{|l|}{ Education } \\
\hline Less than high school diploma & 24,502 & $15.8(15.5-16.2)$ & 10,334 & $12.8(12.4-13.2)$ \\
\hline High school diploma or GED & 82,631 & $29.2(28.9-29.5)$ & 44,364 & $27.0(26.6-27.4)$ \\
\hline Some college or technical school & 77,526 & $31.5(31.1-31.9)$ & 44,601 & $29.7(29.2-30.1)$ \\
\hline College graduate & 91,451 & $22.7(22.5-23.0)$ & 71,318 & $29.1(28.8-29.5)$ \\
\hline \multicolumn{5}{|l|}{ Employment status } \\
\hline Employed & 107,090 & $44.2(43.8-44.6)$ & 116,081 & $68.2(67.8-68.7)$ \\
\hline Unemployed & 12,213 & $6.6(6.4-6.8)$ & 7,594 & $6.1(5.8-6.3)$ \\
\hline Retired & 105,917 & $26.1(25.8-26.4)$ & 25,594 & $7.2(7.0-7.4)$ \\
\hline Unable to work & 29,703 & $11.8(11.5-12.0)$ & 2,364 & $1.6(1.4-1.7)$ \\
\hline Homemaker or student & 20,517 & $10.2(9.9-10.5)$ & 18,339 & $15.0(14.6-15.4)$ \\
\hline \multicolumn{5}{|l|}{ Household income } \\
\hline$<\$ 25,000$ & 77,924 & $30.1(29.7-30.4)$ & 30,419 & $21.7(21.3-22.1)$ \\
\hline$\$ 25,000-<\$ 50,000$ & 64,194 & $21.8(21.5-22.1)$ & 35,578 & $20.1(19.8-20.5)$ \\
\hline $2 \$ 50,000$ & 94,896 & $34.0(33.7-34.4)$ & 82,096 & $42.9(42.5-43.4)$ \\
\hline Missing & 40,743 & $14.1(13.8-14.4)$ & 23,959 & $15.2(14.9-15.6)$ \\
\hline \multicolumn{5}{|l|}{ Marital status } \\
\hline Married & 141,372 & $51.0(50.7-51.4)$ & 99,589 & $50.7(50.2-51.2)$ \\
\hline
\end{tabular}




\begin{tabular}{|c|c|c|c|c|}
\hline \multirow[b]{2}{*}{ Characteristic } & \multicolumn{2}{|c|}{$\begin{array}{l}\text { Unhealthy group } \\
\text { Respondents with at least one of nine } \\
\text { chronic conditions }(n=277,757)\end{array}$} & \multicolumn{2}{|c|}{$\begin{array}{l}\text { Healthy group } \\
\text { Respondents without any of nine chronic } \\
\text { conditions }(n=172,052)\end{array}$} \\
\hline & $\mathbf{N}^{b}$ & $\%(95 \% \mathrm{CI})^{c}$ & $\mathbf{N}^{b}$ & $\%(95 \% \mathrm{CI})^{c}$ \\
\hline Divorced, widowed, separated & 96,678 & $26.8(26.5-27.2)$ & 30,937 & $12.6(12.3-12.9)$ \\
\hline Never married & 32,122 & $18.0(17.6-18.3)$ & 34,350 & $30.7(30.2-31.2)$ \\
\hline Member of an unmarried couple & 5,925 & $3.5(3.3-3.7)$ & 5,714 & $5.0(4.8-5.3)$ \\
\hline \multicolumn{5}{|l|}{ Sleep duration (hours/24 hours) } \\
\hline$\leq$ & 33,729 & $15.0(14.7-15.3)$ & 11,988 & $8.4(8.2-8.7)$ \\
\hline 6 & 59,539 & $23.3(23.0-23.7)$ & 36,406 & $22.5(22.1-22.9)$ \\
\hline 7 & 77,380 & $26.4(26.1-26.8)$ & 59,745 & $32.6(32.2-33.1)$ \\
\hline 8 & 79,829 & $26.3(26.0-26.6)$ & 53,254 & $29.4(29.0-29.9)$ \\
\hline 9 & 15,132 & $4.7(4.5-4.8)$ & 7,130 & $4.2(4.0-4.4)$ \\
\hline$\geq 10$ & 12,148 & $4.3(4.1-4.5)$ & 3,529 & $2.7(2.5-2.9)$ \\
\hline Leisure-time physical activity & 198,162 & $71.9(71.5-72.2)$ & 142,052 & $81.2(80.8-81.6)$ \\
\hline \multicolumn{5}{|l|}{ Body mass index (BMI, kg/m²) } \\
\hline Underweight $(\mathrm{BMI}<18.5)$ & 4,376 & $1.8(1.7-2.0)$ & 2,622 & $2.0(1.9-2.2)$ \\
\hline Normal weight $(\mathrm{BMI}=18.5-24.9)$ & 75,386 & $28.7(28.3-29.1)$ & 62,556 & $39.8(39.3-40.3)$ \\
\hline Overweight $(\mathrm{BMI}=25.0-29.9)$ & 93,560 & $34.5(34.1-34.9)$ & 59,934 & $36.0(35.5-36.5)$ \\
\hline Obesity (BMI 230.0 ) & 89,550 & $35.0(34.6-35.3)$ & 34,916 & $22.1(21.7-22.5)$ \\
\hline \multicolumn{5}{|l|}{ Smoking status } \\
\hline Current smoker & 43,030 & $19.9(19.6-20.2)$ & 21,460 & $14.6(14.3-15.0)$ \\
\hline Former smoker & 90,292 & $30.2(29.8-30.5)$ & 34,776 & $17.9(17.6-18.3)$ \\
\hline Never smoker & 133,940 & $49.9(49.5-50.3)$ & 106,895 & $67.4(67.0-67.9)$ \\
\hline Poor or fair health & 73,921 & $27.9(27.6-28.3)$ & 9,033 & $6.9(6.6-7.1)$ \\
\hline Frequent physical distress & 51,796 & $19.3(19.0-19.6)$ & 5,945 & $4.0(3.8-4.2)$ \\
\hline Frequent mental distress & 37,414 & $17.0(16.7-17.3)$ & 6,850 & $5.3(5.1-5.5)$ \\
\hline Frequent activity limitation & 34,375 & $13.6(13.3-13.9)$ & 3,031 & $2.1(2.0-2.3)$ \\
\hline
\end{tabular}

Abbreviations: CI, confidence interval; GED, General Educational Development high school equivalency diploma

a Nine chronic conditions include coronary heart disease (CHD), stroke, cancer, chronic obstructive pulmonary disease (COPD), diabetes, asthma, arthritis, depression, or chronic kidney disease.

$b_{\text {Unweighted sample of respondents. }}$

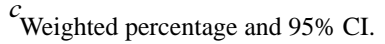

${ }^{d}$ Non-Hispanic. 


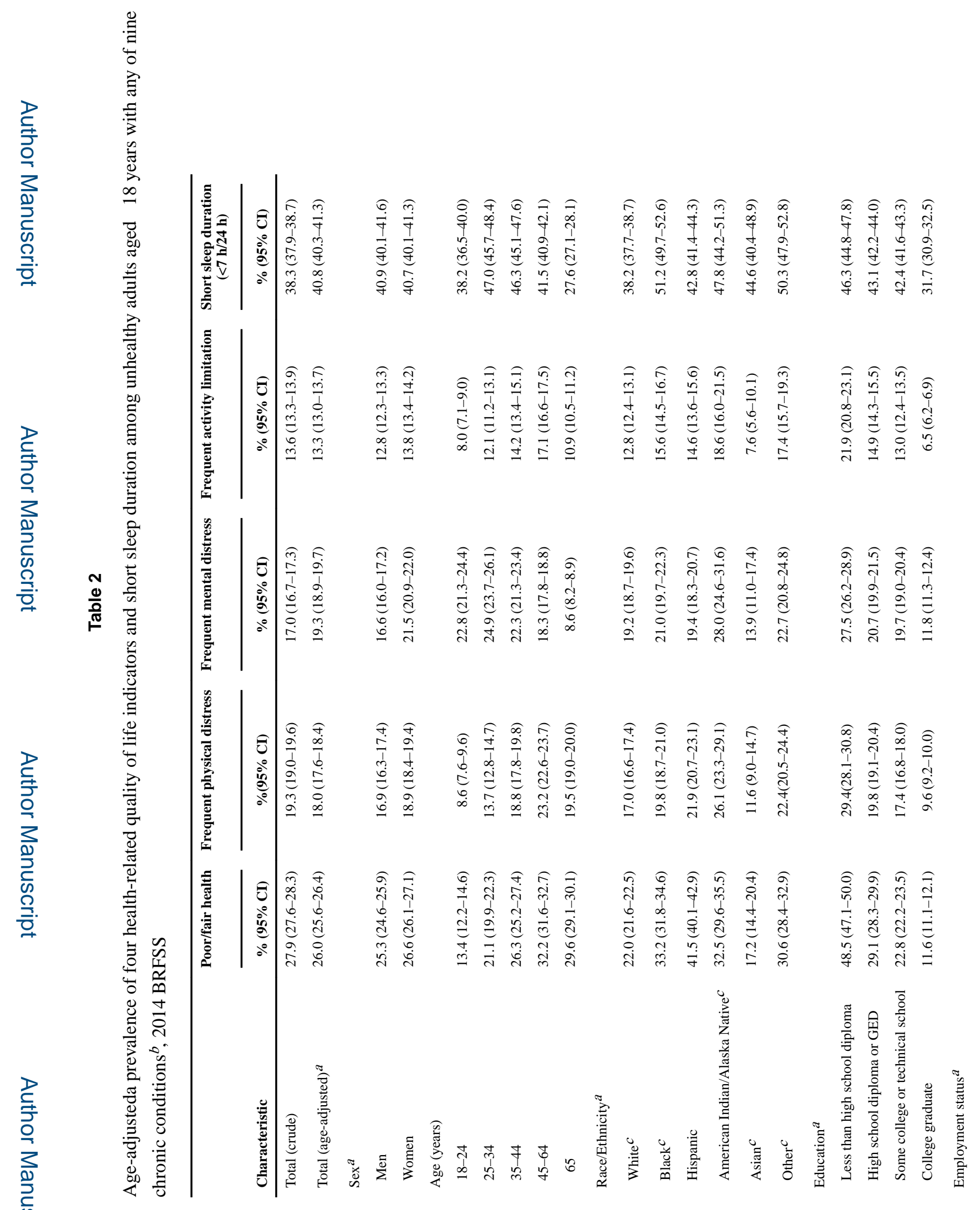

Sleep Health. Author manuscript; available in PMC 2018 July 10. 


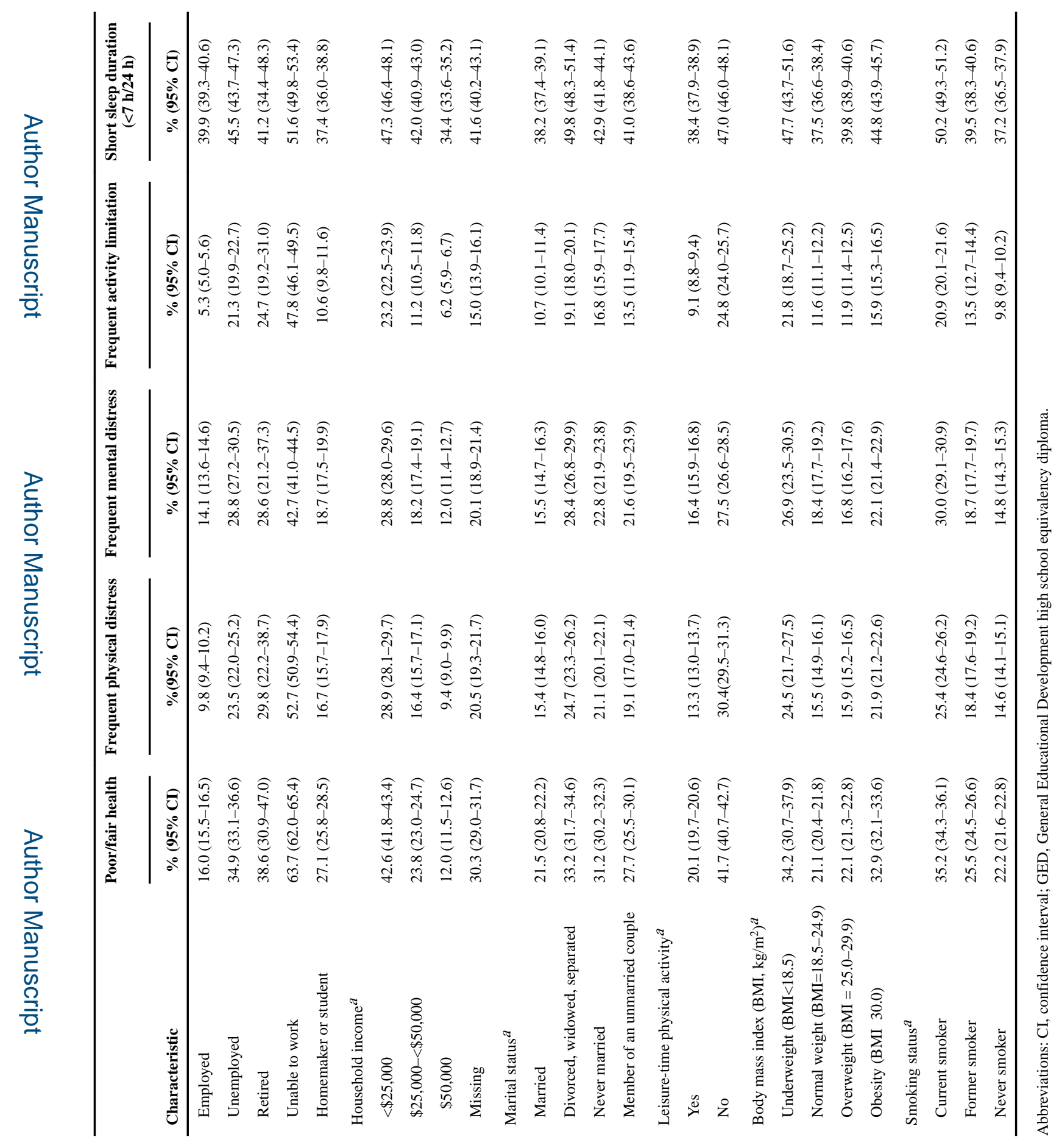

로을

Sleep Health. Author manuscript; available in PMC 2018 July 10. 


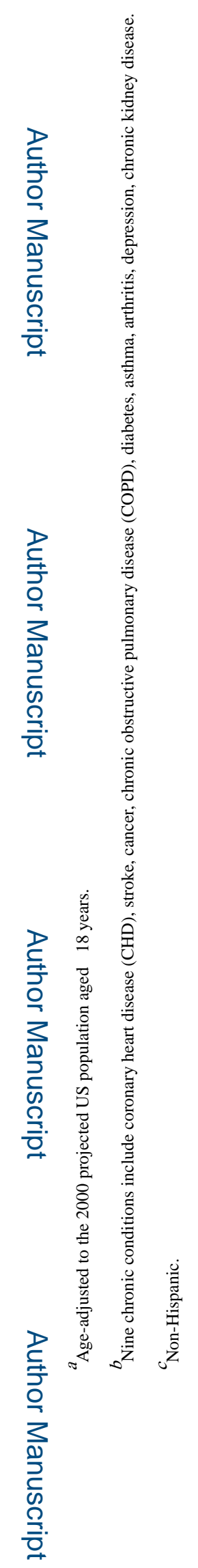

Sleep Health. Author manuscript; available in PMC 2018 July 10. 


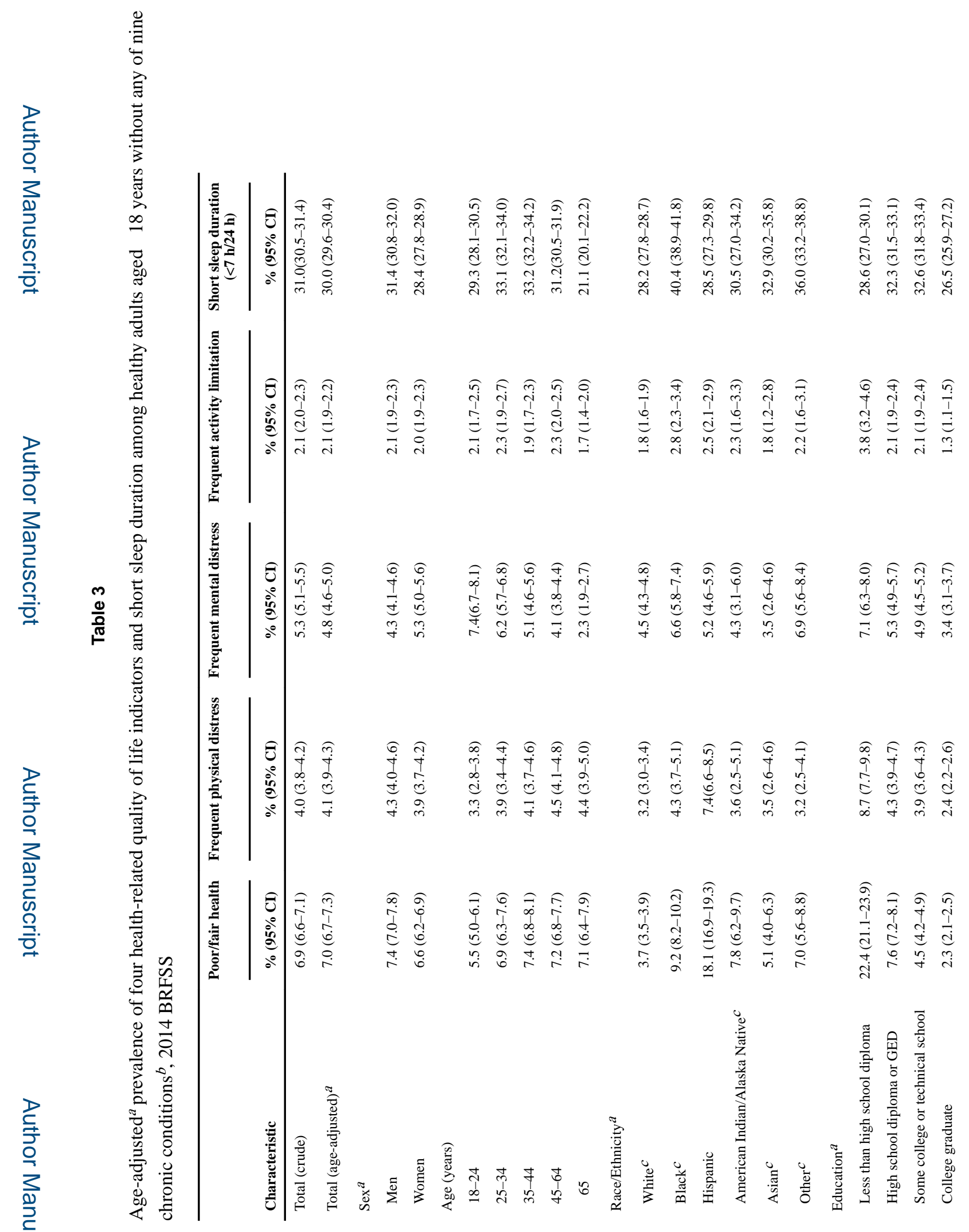

Sleep Health. Author manuscript; available in PMC 2018 July 10. 


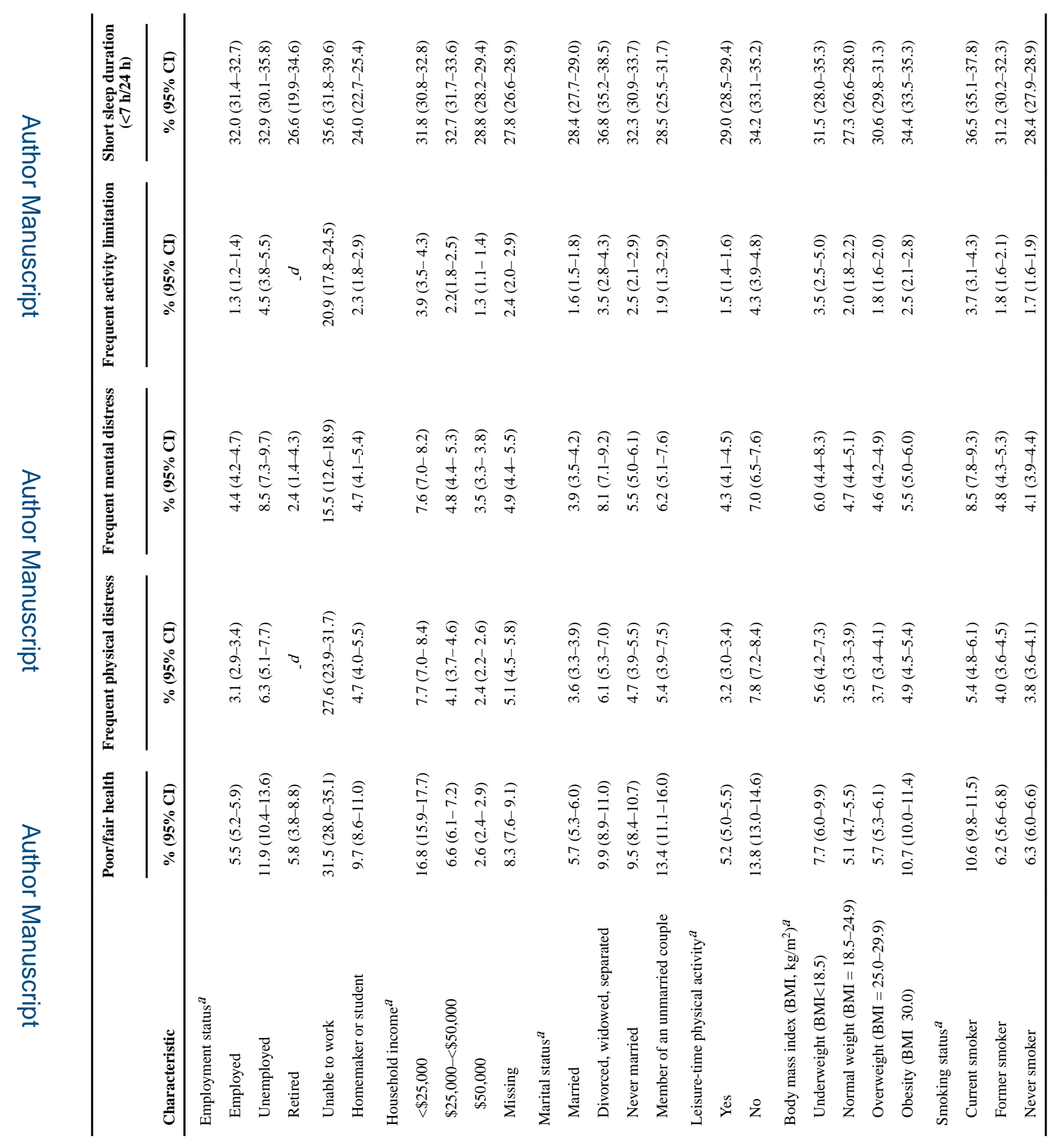

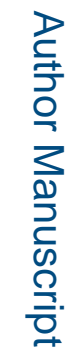




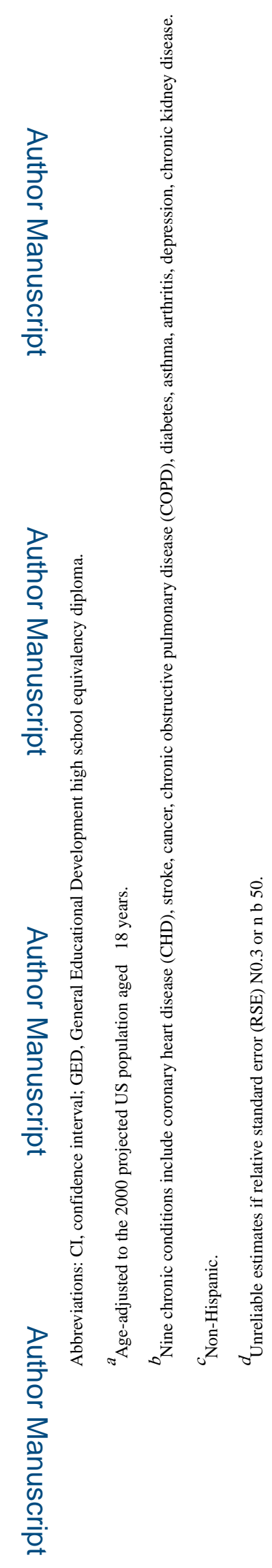

Sleep Health. Author manuscript; available in PMC 2018 July 10. 


\section{Table 4}

Adjusted prevalence and prevalence ratio (PR) and 95\% CI for the risk of poor health-related quality of life by sleep duration among unhealthy and healthy adults ${ }^{a} 2014$ BRFSS

\begin{tabular}{|c|c|c|c|c|}
\hline \multirow[b]{2}{*}{$\begin{array}{l}\text { Sleep duration } \\
\text { (hours in a 24-hour } \\
\text { period) }\end{array}$} & \multicolumn{2}{|c|}{ Unhealthy group } & \multicolumn{2}{|c|}{ Healthy group } \\
\hline & $\begin{array}{c}\text { Age-adjusted } \%(95 \% \\
\text { CI })^{b}\end{array}$ & Adjusted PR $(95 \% \mathrm{CI})^{c}$ & $\begin{array}{c}\text { Age-adjusted } \%(95 \% \\
\text { CI })^{b}\end{array}$ & Adjusted PR $(95 \% \text { CI })^{c}$ \\
\hline \multicolumn{5}{|c|}{ Poor or fair health } \\
\hline$\leq$ & $46.1(45.1-47.2)$ & $1.55(1.49-1.62)$ & $12.0(10.9-13.2)$ & $1.70(1.50-1.94)$ \\
\hline 6 & $29.5(28.8-30.3)$ & $1.25(1.21-1.30)$ & $7.5(6.9-8.1)$ & $1.37(1.22-1.53)$ \\
\hline 7 & $18.3(17.7-18.9)$ & 1.00 (referent) & $4.8(4.4-5.1)$ & 1.00 (referent) \\
\hline 8 & $23.4(22.7-24.0)$ & $1.02(0.98-1.06)$ & $6.6(6.1-7.1)$ & $1.05(0.93-1.17)$ \\
\hline 9 & $26.4(24.9-27.9)$ & $1.15(1.08-1.21)$ & $8.4(6.9-9.9)$ & $1.16(0.94-1.44)$ \\
\hline$\geq 10$ & $46.2(44.4-48.0)$ & $1.43(1.36-1.52)$ & $11.8(9.4-14.2)$ & $1.34(1.04-1.74)$ \\
\hline \multicolumn{5}{|c|}{ Frequent physical distress } \\
\hline 5 & $35.3(34.3-36.3)$ & $1.85(1.76-1.94)$ & $7.4(6.5-8.4)$ & $1.76(1.48-2.10)$ \\
\hline 6 & $19.9(19.2-20.6)$ & $1.34(1.28-1.40)$ & $4.2(3.8-4.7)$ & $1.22(1.05-1.42)$ \\
\hline 7 & $11.6(11.2-12.1)$ & 1.00 (referent) & $3.0(2.7-3.3)$ & 1.00 (referent) \\
\hline 8 & $15.5(15.0-16.1)$ & $1.09(1.04-1.15)$ & $3.6(3.2-3.9)$ & $0.97(0.84-1.13)$ \\
\hline 9 & $18.0(16.7-19.3)$ & $1.16(1.08-1.25)$ & $5.2(4.0-6.4)$ & $1.22(0.94-1.60)$ \\
\hline$\geq 10$ & $34.9(33.2-36.6)$ & $1.66(1.55-1.78)$ & $7.0(4.3-9.6)$ & $1.27(0.85-1.90)$ \\
\hline \multicolumn{5}{|c|}{ Frequent mental distress } \\
\hline 5 & $32.7(31.7-33.7)$ & $2.18(2.05-2.31)$ & $12.9(11.8-14.1)$ & $2.85(2.49-3.27)$ \\
\hline 6 & $18.3(17.6-19.0)$ & $1.47(1.39-1.56)$ & $7.4(6.8-8.0)$ & $1.76(1.55-1.99)$ \\
\hline 7 & $10.1(9.6-10.6)$ & 1.00 (referent) & $3.6(3.3-3.9)$ & 1.00 (referent) \\
\hline 8 & $11.9(11.4-12.4)$ & $1.02(0.96-1.09)$ & $3.3(3.0-3.6)$ & $0.82(0.71-0.94)$ \\
\hline 9 & $14.2(13.0-15.5)$ & $1.16(1.05-1.29)$ & $3.5(2.7-4.3)$ & $0.84(0.64-1.10)$ \\
\hline$\geq 10$ & $28.6(26.9-30.3)$ & $1.76(1.62-1.91)$ & $7.9(5.8-10.0)$ & $1.65(1.20-2.27)$ \\
\hline \multicolumn{5}{|c|}{ Frequent activity limitation } \\
\hline 5 & $27.8(26.8-28.7)$ & $2.16(2.02-2.30)$ & $5.1(4.3-5.9)$ & $2.68(2.13-3.36)$ \\
\hline 6 & $13.8(13.2-14.3)$ & $1.47(1.38-1.56)$ & $2.4(2.1-2.8)$ & $1.68(1.36-2.08)$ \\
\hline 7 & $6.7(6.3-7.0)$ & 1.00 (referent) & $1.2(1.0-1.4)$ & 1.00 (referent) \\
\hline 8 & $10.2(9.7-10.6)$ & $1.15(1.08-1.23)$ & $1.6(1.4-1.8)$ & $1.06(0.86-1.32)$ \\
\hline 9 & $12.5(11.3-13.6)$ & $1.26(1.15-1.39)$ & $2.4(1.5-3.3)$ & $1.30(0.89-1.91)$ \\
\hline$\geq 10$ & $28.9(27.3-30.6)$ & $1.96(1.81-2.14)$ & $6.3(3.6-8.9)$ & $2.35(1.43-3.89)$ \\
\hline
\end{tabular}

Abbreviations: PR-prevalence ratio; CI-confidence interval.

${ }^{a}$ Unhealthy or healthy adults were those with or without any of nine chronic conditions (coronary heart disease (CHD), stroke, cancer, chronic obstructive pulmonary disease (COPD), diabetes, asthma, arthritis, depression, or chronic kidney disease).

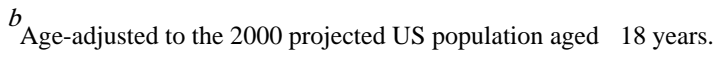

${ }^{c}$ Adjusted PR (prevalence ratio) was obtained from a fully-adjusted logistic regression model, which included sleep duration category, age, sex, race/ethnicity, education, employment status, household income, marital status, leisure-time physical activity, smoking status, and BMI category. 